Harold A. McAlister and William I. Hartkopf (eds.)

\title{
The Fragmentation Mechanism
}

\author{
ALAN P. BOSS \\ Department of Terrestrial Magnetism, Carnegie Institution of Washington, \\ Washington, DC 20015, USA
}

\begin{abstract}
Binary and multiple star systems may form by fragmentation, that is, through break-up of a dense molecular cloud core during the dynamical collapse phase that leads to the formation of protostellar objects. This review concentrates on theoretical models of fragmentation based on numerical hydrodynamical calculations in three spatial dimensions, using both finite-difference and smoothed particle hydrodynamics techniques. A variety of recent results are described, including calculations of the fragmentation of bar-like (prolate) clouds, fragmentation in clouds with initial power-law density and angular velocity distributions, tidally-induced fragmentation, fragmentation in cooling clouds, formation of hierarchical systems, and the dividing line between clouds that fragment and those that appear to form single protostars. A brief comparison of the predicted physical and dynamical properties of the theoretical fragments with the observed properties of main-sequence and pre-main-sequence binary stars lends supports to the hypothesis that fragmentation is the dominant formation mechanism for binary and multiple star systems. The major uncertainties regarding fragmentation are the extent to which precollapse clouds are susceptible to fragmentation, and the degree to which binary fragments undergo orbital decay and possibly mergers through interactions with the enveloping disk.
\end{abstract}

\section{INTRODUCTION}

Fragmentation is a process hypothesized to occur in dense molecular clouds during their self-gravitational collapse to form protostellar objects. Fragmentation occurs when a protostellar cloud breaks up into two or more objects during the dynamic collapse phase (fission, on the other hand, involves the breakup of a rapidly rotating, quasi-equilibrium body). Fragmentation is a leading contender for being the mechanism that is responsible for the formation of the bulk of binary and multiple star systems (see the recent reviews by Pringle 1991, Bodenheimer et al. 1992, Boss 1992). Adams (this Colloquium) describes a closely related mechanism - binary companions resulting from the growth of eccentric density waves in a disk orbiting a protostar, with both components thereafter accreting gas from the collapsing cloud envelope.

Fragmentation was first hypothesized to occur on the basis of Jeans mass arguments (Hoyle 1953) and linear stability analysis of the collapse of a uniform density cloud (Hunter 1962). Because the Jeans mass scales as $M_{J} \propto T^{3 / 2} \rho^{-1 / 2}$, the Jeans mass decreases rapidly during isothermal collapse phases, meaning that smaller and smaller objects can become gravitationally bound and could become distinct entities. Protostellar collapse itself has not yet been conclusively shown to occur in low-mass young stellar objects, so perhaps it is too soon to hope for observational evidence for fragmentation. However, this situation may change as $\mathrm{mm}$ - and sub-mm wave interferometric arrays grow in size and sensitivity and as the next generation of optical/infrared telescopes becomes operational. In the meantime, much of our understanding of whether fragmentation can occur must come from theoretical modeling. This review will summarize 
our current theoretical understanding of the fragmentation mechanism.

Because of the intrinsically nonlinear, three-dimensional, non-stationary nature of the fragmentation process, our analytical understanding is still very rudimentary, being limited either to simple arguments such as the Jeans mass or to empirically-derived relations (e.g., the $\alpha_{i} \beta_{i}<0.12$ constraint of Miyama et al. 1984; see section 2). Essentially all of our knowledge of fragmentation has come from numerical hydrodynamics calculations. Three-dimensional hydrodynamics calculations were pioneered in the late 1970s, but the development of substantially improved numerical codes has led recently to a rebirth of interest in fragmentation calculations. The next two sections describe these new fragmentation calculations, followed by a brief comparison of theoretical fragment properties with those of observed binary and multiple systems.

\section{INITIALLY UNIFORM DENSITY CLOUDS}

Larson (1972) was first to study the collapse of a rotating, axisymmetric (2D) cloud. Larson found that a rapidly rotating cloud could collapse to form a ring, which he recognized might very well fragment in a three dimensional (3D) calculation, thereby explaining the formation of binary star systems. Bodenheimer (1978) used similar 2D calculations to suggest the possibility of hierarchical fragmentation through the formation of a series of rings and fragments of decreasing sizes. These 2D calculations assumed a highly idealized, spherical initial cloud with uniform density and rotation, assumptions which have often been adopted in subsequent $3 \mathrm{D}$ models.

3D hydrodynamical calculations have shown that not all initially uniform density clouds undergo fragmentation during collapse, and that the manner in which fragmentation occurs (e.g., directly, or through an intermediate ring phase) depends on the initial cloud configuration as well. Hence one basic goal for 3D numerical calculations has been to examine the possible parameter space for initial clouds as thoroughly as possible (e.g., Bodenheimer et al. 1980). Figure 1 shows the results of all published 3D models of the collapse of initially uniform density, isothermal clouds, calculated with either finite-difference or smoothed particle hydrodynamics (SPH) codes (references in Boss 1990 and below). A remarkable amount of agreement is evident about the three possible endstates for collapse. Uniform density, isothermal clouds can expand to form equilibrium Bonnor-Ebert ellipsoids, collapse to form single protostars, or collapse and undergo fragmentation into two or more objects, depending on the initial values of $\alpha_{i}=E_{\text {thermal }} /\left|E_{\text {gravity }}\right|$ and $\beta_{i}=E_{\text {rotational }} /\left|E_{\text {gravity }}\right|$ for the cloud. The empirical criteria (Figure 1) of Miyama et al. (1984) are fairly good at delineating these three outcomes, with the exception of the low $\beta_{i}$ regime. Note the 'single' result for the $\alpha_{i}=0.55, \beta_{i}=0.02$ model (Bodenheimer et al. 1980), a model which clearly meets the $\alpha_{i} \beta_{i}<0.12$ criterion for fragmentation. Clouds with relatively high $\alpha_{i}$ and low $\beta_{i}$ do not appear to undergo fragmentation, and so the empirical criterion does not apply in this regime.

In extreme cases, fragmentation can be primarily thermally, rotationally, or externally driven. Fragments can grow directly from the initial density perturbation for low $\alpha_{i}$ clouds in the first case, or in the second case fragments can grow from the rotationally supported, intermediate configurations (rings or 


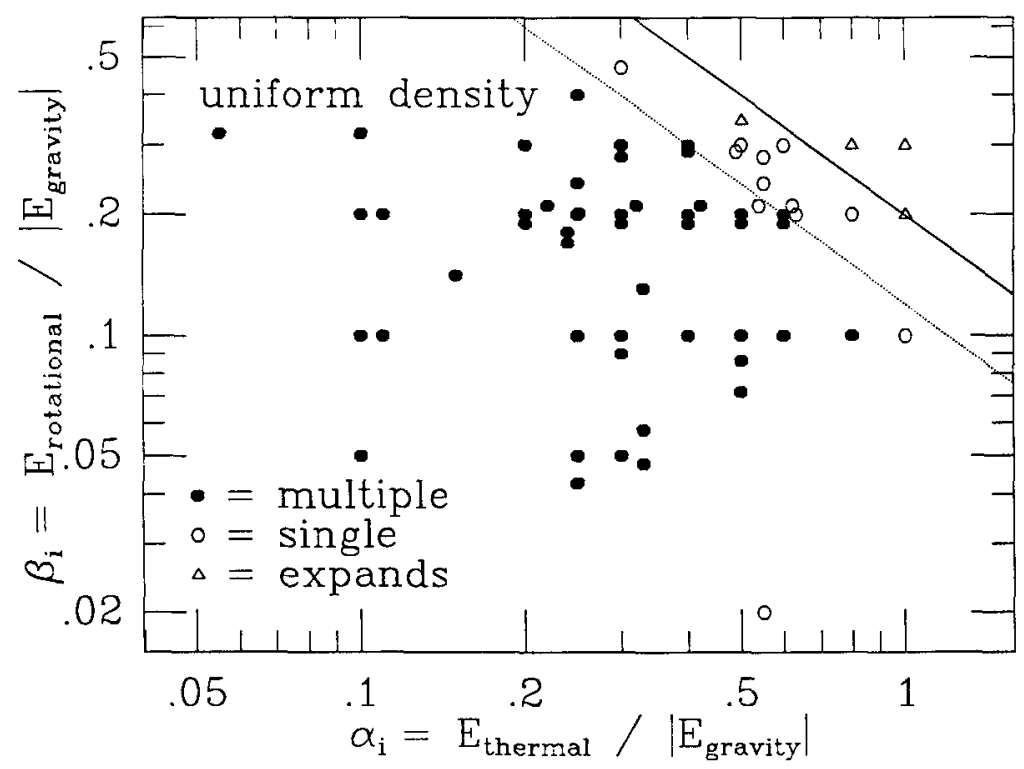

FIGURE 1. Results of all 3D calculations of the isothermal collapse of initially uniform density clouds. Solid line is the criterion $\left(\alpha_{i} \beta_{i}>0.2\right)$ for expansion and the dotted line is the criterion for fragmentation $\left(\alpha_{i} \beta_{i}<0.12\right)$ proposed by Miyama et al. (1984).

disks) formed in high $\beta_{i}$ clouds. A third possibility is fragmentation that is induced by external effects such as tidal perturbations. In less extreme cases, fragmentation may involve varying degrees of all three effects.

An interesting example of combined thermally and rotationally driven fragmentation occurs in an SPH model calculated by Monaghan \& Lattanzio (1991). They first verified that an initially uniform density, isothermal cloud with $\alpha_{i}=$ 0.3 and $\beta_{i}=0.47$ collapsed and formed a stable disk, i.e. a single protostar, consistent with the empirical criterion for intermediate values of the quantity $\alpha_{i} \beta_{i}$. The final disk was both thermally and rotationally supported. Monaghan \& Lattanzio then recalculated the same model, only allowing for cooling of the cloud from its initial temperature of $70 \mathrm{~K}$; the cooling rate was chosen to be appropriate for radiative losses from molecular lines. By the time the cloud had cooled to $5 \mathrm{~K}$, the formerly stable cloud had collapsed to form a disk that broke up into a number of string-like filaments, with growing 'knots' located at the intersections of the strings. Six knots (with roughly equal spacings) had formed by the end of the calculation. In this case the thermal cooling allowed the fragmentation to take place, but the large number of fragments was made possible by the rotationally supported disk.

In addition to starting from uniform density, it has been traditional to calculate the collapse of initially spherical clouds - the idea being to start from as symmetrical an initial state as possible, in order to make a conservative estimate about the prevalence of fragmentation. However, nature may not be so conservative. In fact, observational evidence for bar-like (prolate) molecular cloud cores 
has been presented by Myers et al. (1991). These observations suggest that collapse calculations should start from highly nonaxisymmetric initial clouds. Bastien, Bonnell, and their colleagues have been vigorously studying the fragmentation of initially cylindrical clouds with aspect ratios similar to those of the Myers et al. cloud cores. Bastien et al. (1991) calculated the fragmentation of isothermal, non-rotating cylinders, using both finite-difference and SPH codes. They found that as $\alpha_{i}$ was lowered, the cylinders fragmented into more and more objects. However, they found that the number of fragments saturated at a value of $\sim 2 L / D$, where $L$ is the length and $D$ is the diameter of the initial cylinder $(10 \geq L / D \geq 2)$, even for very low values of $\alpha_{i}(<0.08)$. A linear stability analysis for infinite cylinders predicts ever-increasing numbers of fragments as the thermal energy is lowered, as does the Jeans mass argument, which is itself based on the linear stability of an infinite homogeneous medium. These numerical results help illustrate the limited applicability of linearized analysis to the fragmentation of realistic configurations. These models also are clear examples of thermally driven fragmentation.

Bonnell et al. (1991) then used their SPH code to study the fragmentation of rotating, isothermal cylinders, with $L / D=2$ or $3, \alpha_{i}=0.25,0.33$, or 0.5 , and $\beta_{i}=0.01$ to 0.13 . Each of these cylinders collapsed and fragmented directly into a binary or multiple (3-, 4-, or 5-body) protostellar system. Lower initial values of $\alpha_{i}$ produced larger numbers of fragments - a sign of thermally driven fragmentation, in spite of the presence of rotation. The mass ratios of the binary fragments ranged from $\sim 0.2$ to 0.8 , with most of the cloud mass having been accreted by the end of the calculations. Maximum separations of the fragments were on the order of $10^{3}$ to $10^{4} \mathrm{AU}$. Orbital eccentricities for the binary fragments were large $(e \sim 0.4$ to 0.8$)$, because fragmentation did not occur through an intermediate centrifugally-supported disk.

Binary fragments with similar properties have been found in finite-difference models of the collapse of uniform density, isothermal clouds. Boss (1992) found that binaries formed by fragmentation generally have large initial eccentricities ( $e \sim 0.3$ to 0.9 ), and initial mass ratios ranging from $\sim 0.1$ to 1 , though in these models most of the cloud envelope has not been accreted by the binary protostars and significant further accretion of mass is to be expected.

Bonnell et al. (1992) modeled the fragmentation of isothermal cylindrical clouds with rotation about arbitrary rotation axes. For clouds that are primarily rotating about an axis perpendicular to the long axis of the cylinder, binary fragments grow at the ends of the cylinder (as in Bonnell et al. 1991), only now the fragments and their surrounding disks are not coplanar. As the amount of rotation around the axis parallel to the cylinder's long axis is increased, the binary fragments are delayed in forming and fall closer together, where they can experience significant mutual tidal forces and mass transfer between the fragments. For low $\alpha_{i}$, the binary fragments may undergo sub-fragmentation and form hierarchical multiple systems. Tidal interactions with the companion system also appeared to lead to sub-fragmentation.

The effect of tidal interactions with external objects was studied by Felice \& Sigalotti (1991) and by Sigalotti \& Klapp (1992), using a finite-difference code developed by Sigalotti (1990). Sigalotti's (1990) code was the first finitedifference code to relax the assumption of symmetry above and below the equatorial plane. The code is thus able to study the tidal effects of nearby protostars 

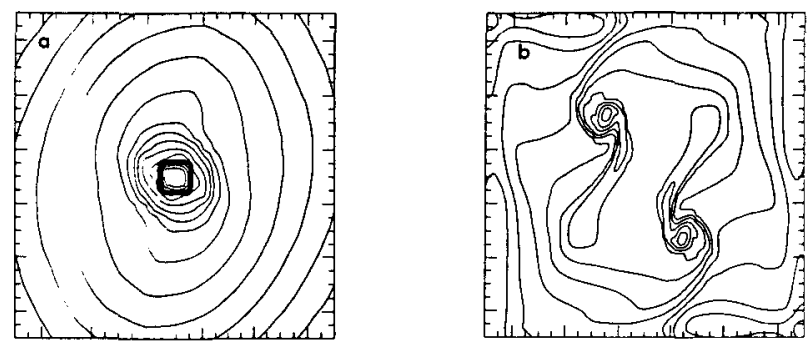

FIGURE 2. Results of two models of 3D protostellar collapse (Myhill \& Kaula 1992). Equatorial (log) density contours are shown on a scale of about $5000 \mathrm{AU}$. Both models started from $\rho_{i} \propto r^{-1}$ with $\alpha_{i}=0.16$ and $\beta_{i}=0.17$, but (a) had $\Omega_{i} \propto c$ while (b) had $\Omega_{i} \propto r^{-2 / 3}$.

with arbitrary locations with respect to the spherical cloud in question. Felice \& Sigalotti (1991) found that very strong tidal forces (from 3 more or less randomly placed ob jects at distances of several cloud radii) could disrupt the cloud's collapse and thereby prevent fragmentation, whereas more moderate tidal forces enhanced the tendency for clouds to fragment, compared to clouds without any tidal perturbations. The externally driven fragmentation in these models tended to occur through an intermediate bar-shaped configuration resulting from the dominance of that term in an expansion of the tidal potential. Sigalotti \& Klapp (1992) extended these studies to include the collapse of clouds with adiabatic thermodynamics, simulating the nonisothermal regime of protostellar evolution $\left(\rho>10^{-13} \mathrm{~g} \mathrm{~cm}^{-3}\right)$. The clouds were subjected to tidal forces from companion objects on elliptical trajectories. They found that tidal forces in this phase of evolution did not have much effect on the final outcome, that is, whether binary sub-fragmentation occurred or a near-equilibrium ellipsoid formed. Evidently externally driven fragmentation becomes ineffective in the nonisothermal regime.

\section{INITIALLY NONUNIFORM DENSITY CLOUDS}

All of the work discussed so far involved the collapse of initially uniform density clouds. The assumption of initially uniform density clearly errs on the side of uniformity - yet isothermal clouds must be centrally condensed if thermal pressure is to play a role in supporting the precollapse cloud. In fact, observations of dense molecular cloud cores (e.g., Zhou et al. 1990) are consistent with powerlaw density profiles ( $\rho \propto r^{-n}$, with $n \approx 1.5$ to 2 ) in the cloud envelopes on scales of $0.02 \mathrm{pc}$ to $0.2 \mathrm{pc}$. These observations suggest the applicability of the opposite extreme for the initial density profile - the 'singular isothermal sphere', with $\rho \propto r^{-2}$, for which a similarity solution exists (Shu 1977). This interpretation is complicated by the fact that even the extreme case of an initially uniform density cloud collapses to produce an envelope with a power-law density profile. At any rate, singular isothermal spheres and uniform density clouds bracket the 
possible range of initial density profiles.

Myhill \& Kaula (1992) studied the collapse of clouds (Figure 2) with powerlaw initial density profiles ( $n=1$ or 2 ) and varied initial angular velocity profiles $\left(\Omega_{i} \propto \rho^{2 / 3}\right.$, appropriate for prior contraction with conserved angular momentum, and $\Omega_{i}=c$, appropriate for contraction with constant angular velocity). For the case of $n=1$ or 2 and $\Omega_{i}=c$, they found that the initial central concentration of mass inherent in power-law density profiles was stable to fragmentation, consistent with the results of Boss (1987) for $n=1$. However, Myhill \& Kaula (1992) found that when the clouds were initially differentially rotating such that the central regions were rotating much more rapidly than the outer regions, fragmentation could occur for either the $n=1$ or 2 initial density profile, all for clouds with the same values of $\alpha_{i}=0.16$ and $\beta_{i}=0.17$. Myhill \& Kaula's models clearly indicate that rotationally driven fragmentation can occur in power-law initial density clouds, provided that these clouds are differentially rotating with significant rotational energy.

A power-law density profile is not appropriate for the entire radial extent of a precollapse cloud, if for no other reason than the existence of a density singularity at the cloud center. At some radius, the density profile must turn over and tend toward a finite central density - a maximum density ultimately to be determined by observations of the densest regions of cold cloud cores that do not appear to contain embedded protostellar objects. At the present, maximum inferred densities are on the order of $\sim 10^{-18} \mathrm{~g} \mathrm{~cm}^{-3}$, though this value is influenced by the molecule being observed, and even higher density regions undoubtedly exist in precollapse clouds. Another approach to defining the structure of precollapse clouds is to consider theoretical models of magnetically supported, rotating clouds contracting under the influence of ambipolar diffusion (Tomisaka et al. 1990). Magnetic fields appear to be quite important for molecular cloud support, at least in diffuse cloud phases. The models of Tomisaka et al. show that magnetically supported, rotating clouds have power-law density profiles in their envelopes, and that these profiles flatten out toward the centers of the clouds.

The foregoing considerations suggest that a Gaussian $\left(\rho_{i} \propto e^{-(r / R)^{2}}\right)$ initial density profile might be an appropriate choice for precollapse clouds. This initial density profile was used in the 3D nonisothermal models of Boss (1987), who found that thermally driven fragmentation could still occur (though only for slightly smaller values of $\alpha_{i}$ than in the uniform density case), when the initial central density was 20 times the initial density at the outer edge of the cloud. Boss (1991) recalculated a series of Gaussian profile models from the Boss (1987) paper with an improved 3D finite-difference code (Boss \& Myhill 1992) and found an enhanced degree of fragmentation in the new models. A sequence of models with $\beta_{i}=0.16$ and varied $\alpha_{i}=0.06,0.13,0.26$, and 0.4 all experienced binary fragmentation. Furthermore, the binary fragments in the model with $\alpha_{i}=0.26$ sub-fragmented, producing a hierarchical multiple system with four protostellar cores. Because of the formation of intermediate disk configurations in the models with $\alpha_{i}=0.26$ and 0.4 , fragmentation in these models was primarily rotationally driven, whereas for the lower $\alpha_{i}$ models fragmentation was primarily thermally driven and occurred through intermediate bar configurations. 
The collapse of identically centrally condensed clouds but with much lower initial rotation rates $\left(\beta_{i} \sim 0.01,0.0001\right)$ has also been calculated with the new code (Boss 1992, in preparation). Clouds with slow rotation soon reach densities of $\sim 10^{-13} \mathrm{~g} \mathrm{~cm}^{-3}$ where the central regions are optically thick in the infrared, requiring inclusion of the effects of radiative transfer. These models have initially Gaussian radial density profiles, modified into prolate spheroids with axis ratios of $2: 1$ or $1.5: 1$. The results of a number of models with 1.5:1 axis ratios are shown in Figure 3. As in the case of initially uniform density clouds, three different outcomes result - expansion, collapse to form a single object, or collapse and fragmentation. By comparing with Figure 1, we can see that even these moderately centrally condensed clouds require significantly lower values of $\alpha_{i}$ in order for collapse and fragmentation to occur. For example, for $\beta_{i} \approx 0.1$, the critical value of $\alpha_{i}$ for fragmentation is about 0.9 for uniform initial density and about 0.3 for these centrally condensed clouds. The absence of any strong dependence on $\beta_{i}$ in Figure 3 implies that fragmentation is primarily thermally driven in these models. Figure 3 also shows that the empirical criteria derived for uniform density clouds do not apply to these centrally-condensed clouds.

The calculations discussed so far refer to the possibility of fragmentation during the first dynamic collapse phase of protostellar evolution, prior to the formation of the first, thermally supported, outer core. Boss (1989) has calculated 3D models of the second dynamic collapse phase, after molecular hydrogen dissociates. This phase forms the final protostellar core. Boss (1989) found that even in the collapse of an initially rapidly rotating first core, fragmentation was unable to produce a stable binary system. The rotationally driven fragmentation that did occur was effectively countered by the growth of trailing spiral arms which quickly absorbed the angular momentum of the binary fragments and led to their orbital merger. The prospects for thermally driven fragmentation during the second collapse phase are equally dismal, because of the large amount of thermal energy in the first core. Given that externally driven fragmentation is unlikely to be effective during the second collapse phase (e.g., Sigalotti \& Klapp 1992), fragmentation appears to be possible only during the first collapse phase.

\section{COMPARISON WITH BINARY STAR PROPERTIES}

Detailed comparisons of observed binary star properties and the 'predictions' of various theoretical formation mechanisms are given by Bodenheimer et al. (1992) and Boss (1992). Here we simply summarize the salient physical and dynamical properties of protostellar cores formed in fragmentation calculations.

- System type: Besides binary systems, fragments have been produced in Trapezium-like systems ( 3 or more roughly equally spaced fragments) and in hierarchical systems (e.g., 4 fragments distributed in 2 short period and 1 long period system).

- Orbital eccentricity: A wide range in initial orbital eccentricities ( $\sim 0.1$ to $\sim 1$ ) has been found. Significant evolution of the initial eccentricity may result from interactions (gravitation, accretion) of the fragments with the parent cloud and disk (see also Clarke, this Colloquium). 


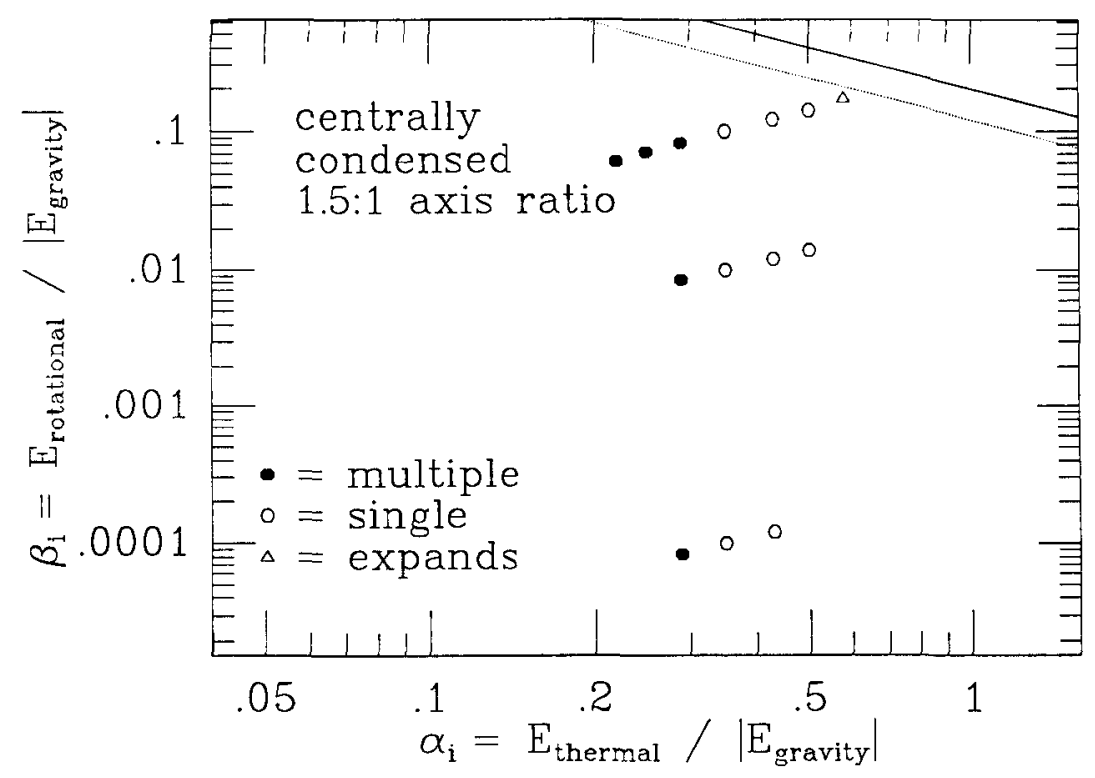

FIGURE 3. Results of the nonisothermal collapse of initially centrally condensed clouds with prolate configurations (Boss, 1992, in preparation). The two lines have the same meaning as in Figure 1.

- Orbital separations: Relatively close $(\sim \mathrm{AU})$ to wide $(\sim 0.1 \mathrm{pc})$ binary separations have been obtained, though again these orbital separations may be subject to significant further evolution. The formation of very close (near-contact) systems may require the partial orbital decay of close systems.

- Spin-orbit and orbit-orbit alignments: The degree of alignment of binary star spin axes and orbital angular momentum vectors will be strongly dependent on the degree of uniformity in the precollapse cloud core's angular momentum distribution. An initially elongated cloud with an asymmetric rotation axis can produce fragments with non-coplanar disks.

- Mass ratios: Secondary/primary mass ratios ranging from $q \sim 0.1$ to 1 have been produced. Further accretion of gas may alter this ratio.

- Frequency: The expected frequency distribution will depend on the properties of the ensemble of precollapse clouds, which unfortunately is not sufficiently well-determined at present to allow firm predictions about the prevalence of fragmentation to be made. Figures 1 and 3 illustrate the range of initial cloud parameter space that results in fragmentation.

- Timing: Fragmentation occurs during the protostellar collapse phase, so binary protostars should be observable. By definition, fragmentation occurs prior to the appearance of optically-visible pre-main-sequence stars. 


\section{CONCLUSIONS}

Fragmentation into binary and multiple protostellar systems can occur in precollapse clouds with suitable characteristics. Fragmentation can be primarily thermally, rotationally, or externally driven. Cloud characteristics that generally are favorable for fragmentation include non-singular density profiles, a low degree of thermal pressure and magnetic field support, significant rotation, and the presence of external perturbations such as tidal forces. Fragmentation can occur during the first (isothermal and early nonisothermal) phase of protostellar collapse, but apparently not during the second collapse phase.

The dynamical properties of the systems of protostellar cores formed by fragmentation appear to be in agreement with the ranges of these properties as determined by observations of binary and multiple pre-main-sequence and main-sequence stars. The extent to which these properties evolve during the subsequent evolution of protostellar cores into pre-main-sequence stars is an important question that is just now beginning to be addressed.

\section{ACKNOWLEDGEMENTS}

This work has been partially supported by NSF grant AST 88-17334.

\section{REFERENCES}

Bastien, P., Arcoragi, J.-P., Benz, W., Bonnell, I., \& Martel, H. 1991, ApJ, 378, 255 Bodenheimer, P. 1978, ApJ, 224, 488

Bodenheimer, P., Ruzmaikina, T., \& Mathieu, R.D. 1992, in Protostars \& Planets III, ed. E. H. Levy, J. I. Lunine, and M. S. Matthews (Tucson, University of Arizona), in press

Bodenheimer, P., Tohline, J. E., \& Black, D. C. 1980, ApJ, 242, 209

Bonnell, I., Arcoragi, J.-P., Martel, H., \& Bastien, P. 1992, A pJ, submitted

Bonnell, I., Martel, H., Bastien, P., Arcoragi, J.-P., \& Benz, W. 1991, ApJ, 377, 553

Boss, A.P. 1987, $A p J, 319,149$

Boss, A.P. $1989, A p J, 346,336$

Boss, A.P. 1990, in Physical Processes in Fragmentation and Star Formation, ed. R. Capuzzo-Dolcetta, C. Chiosi, and A. DiFazio (Dordrecht, Kluwer), p. 279

Boss, A.P. 1991, Nature, 351, 298

Boss, A.P. 1992, in Close Binaries, ed. J. Sahade, G. McCluskey, and Y. Kondo (Dordrecht, Kluwer), in press

Boss, A.P. \& Myhill, E. A. 1992, ApJS, submitted

Felice, F.D. \& Sigalotti, L. D. G. 1991, MNRAS, 249, 248

Hoyle, F. 1953, ApJ, 118, 513

Hunter, C. $1962, A p J, 135,594$

Larson, R.B. $1972, M N R A S, 156,437$

Miyama, S.M., Hayashi, C., \& Narita, S. 1984, $A p J, 279,621$

Monaghan, J.J. \& Lattanzio, J.C. 1991, ApJ, 375, 177

Myers, P.C., Fuller, G. A., Goodman, A. A., \& Benson, P. J. 1991, ApJ, 376, 551

Myhill, E.A. \& Kaula, W.M. 1992, ApJ, 386, 578

Pringle, J.E. 1991, in The Physics of Star Formation and Early Stellar Evolution, ed. C. J. Lada and N. D. Kylafis (Dordrecht, Kluwer), p. 437

Shu, F.H. 1977, $A p J, 214,488$

Sigalotti, L.D.G. 1990, MNRAS, 246, 243 
Sigalotti, L.D.G. \& Klapp, J. 1992, MNRAS, 254, 111

Tomisaka, K., Ikeuchi, S., \& Nakamura, T. 1990, ApJ, 362, 202

Zhou, S., Evans, N.J. II, Butner, H.M., Kutner, M.L., Leung, C.M., \& Mundy, L.G. 1990, ApJ $, 363,168$

\section{DISCUSSION}

ZINNECKER: To what extent are the binary components that form in your 3D calculations coeval? Is there any way to explain the presence of "infrared companions" by non-coeval fragmentation?

BOSS: Given that most of the fragmentation must occur within the time scale of the free-fall time of the parent cloud; non-coevality in the simplest scenario should not extend beyond about $10^{5}$ to $10^{6} \mathrm{yr}$ for solar type stars. Smaller mass fragments will begin their own collapse from lower densities than more massive fragments (in general), making their timescales for evolution longer than their more massive siblings. Companions which are strongly non-coeval may thus require some additional physics for their expanation, e.g., cluster interactions and capture perhaps, or a collision with another molecular cloud core and subsequent accretion of additional gas.

MATHIEU: First, if I understand, the binaries that you are forming are embedded within substantial disks. Would you expect the binaries which you have produced to have both circumstellar and circumbinary disks by, say, the stellar birthline? Second, can fragmentation produce non-coplanar multiple systems?

BOSS: Yes, binaries formed by fragmentation are surrounded from birth by significant disk material. Some of this disk matter may well persist until the protostar becomes optically visible - precise answer cannot be given because this question has not yet been addressed by calculations. Ian Bonnell and Pierre Bastien have recently calculated the collapse of elongated cylindrical clouds rotating about asymmetrically-oriented axes and shown that non-coplanar fragments and disks can result.

HENRY: Do you have a specific wish list for what you'd like observers to find out so that you can limit your parameter space?

BOSS: If observers could find evidence of the existence of binary protostellar objects, which must exist if fragmentation is operative, that would tell us if we even need to consider fragmentation at all. Observations of precollapse clouds are critical for determining the outcome of the subsequent collapse. Key quantities for these clouds are the density and angular velocity profiles, degree of asymmetry in the cloud, amount of magnetic field support, and total energy balance: gravitational, thermal, rotational and magnetic.

MAZEH: Can you tell us what observed distributions fragmentation cannot produce?

BOSS: I believe that fragmentation is capable of reproducing at least the range of binary star properties for all quantities that I am aware of, e.g., mass ratios, separations, eccentricities, system types, etc. The real unanswered question is whether precollapse clouds have suitable properties to allow fragmentation to 
even occur at all, and if so to produce distributions of properties similar to those observed.

POVEDA: I have two questions: have you determined the mass spectrum of the fragmentation, and do you find the two mass distributions that Abt has shown to be dependent on the separation of the binaries?

BOSS: In order to make a rigorous prediction of the frequency distribution of the properties of protostellar cores formed by fragmentation, one must know the distribution of properties of precollapse molecular cloud cores. Unfortunately, while we know a fair amount about dense cloud cores, we do not yet have a firm understanding of the properties of precollapse clouds, i.e. of their density and angular velocity profiles, degree of magnetic support, and so on. It would be interesting to use the observed properties of binary star separations and mass distributions to try to infer the properties of the precollapse cloud population that would be needed to account for their formation by fragmentation. 